\title{
POSITIVE SOLUTIONS AND MULTIPLE SOLUTIONS AT NON-RESONANCE, RESONANCE AND NEAR RESONANCE FOR HEMIVARIATIONAL INEQUALITIES WITH $p$-LAPLACIAN
}

\author{
D. MOTREANU, V. V. MOTREANU, AND N. S. PAPAGEORGIOU
}

\begin{abstract}
In this paper we study eigenvalue problems for hemivariational inequalities driven by the $p$-Laplacian differential operator. We prove the existence of positive smooth solutions for both non-resonant and resonant problems at the principal eigenvalue of the negative $p$-Laplacian with homogeneous Dirichlet boundary condition. We also examine problems which are near resonance both from the left and from the right of the principal eigenvalue. For nearly resonant from the right problems we also prove a multiplicity result.
\end{abstract}

\section{INTRODUCTION}

In this paper we study the following nonlinear elliptic eigenvalue problem:

$$
\left\{\begin{array}{l}
-\operatorname{div}\left(\|D x(z)\|^{p-2} D x(z)\right)-\lambda|x(z)|^{p-2} x(z) \in \partial j(z, x(z)) \text { a.e. on } Z, \\
\left.x\right|_{\partial Z}=0,1<p<+\infty .
\end{array}\right.
$$

Here $Z \subset \mathbb{R}^{N}$ is a bounded domain with a $C^{1, \alpha}$-boundary $\partial Z(0<\alpha<1), j(z, x)$ is a measurable function which is locally Lipschitz in the $x \in \mathbb{R}$ variable and $\partial j(z, x)$ stands for the generalized subdifferential of $x \mapsto j(z, x)$ in the sense of Clarke [5]. The solutions of (1.1) are sought in $W_{0}^{1, p}(Z)$.

Problem (1.1) belongs to the class of hemivariational inequalities that are new types of variational expressions arising if one considers more realistic mechanical laws of multivalued and nonmonotone nature (see [17], [15]). The corresponding energy (Euler) functionals are nonsmooth and nonconvex. Eigenvalue problems as (1.1) enter in the stability analysis of structures whose equilibrium position is characterized by a hemivariational inequality. The mathematical theory of eigenvalue problems for hemivariational inequalities was studied in [3], [6], [9], [10], [13], [14] for semilinear problems (i.e., $p=2$ ), whereas nonlinear eigenvalue problems driven by the $p$-Laplacian were investigated in [7]. None of the aforementioned works addressed the problem of existence of positive solutions.

Theorems on the existence of positive and multiple solutions for hemivariational inequalities involving the $p$-Laplacian have recently been presented in [16]. In this paper we focus on the existence of positive solutions and nontrivial multiple solutions to the eigenvalue problem (1.1) under assumptions different than those of [16], including growth conditions of rate bigger than $p$ for the nonsmooth potential $j(z, x)$. Specifically, denoting by $\lambda_{1}$ the principal (or first) eigenvalue of the negative

Received by the editors February 14, 2006.

2000 Mathematics Subject Classification. Primary 35J20, 35R70; Secondary 35J60, 35J85.

Key words and phrases. Hemivariational inequality, eigenvalue problem, resonance. 
$p$-Laplacian $\left(-\Delta_{p}, W_{0}^{1, p}(Z)\right)$ we are able to show the existence of positive solutions in the non-resonant case $\lambda<\lambda_{1}$ and in the resonant case $\lambda=\lambda_{1}$. First, under some natural assumptions set up around a weaker version of the Ambrosetti-Rabinowitz condition, it is shown that for $\lambda<\lambda_{1}$ problem (1.1) has a positive solution. If $p=2$, by slightly strengthening the assumptions a converse result is provided showing that generally in this setting we cannot relax the non-resonant condition $\lambda<\lambda_{1}$ to have positive solutions for problem (1.1). However, we show that keeping the generalized nonsmooth version of Ambrosetti-Rabinowitz type hypothesis but imposing a different assumption on the generalized gradient with respect to $x \in \mathbb{R}$ of the nonsmooth potential $j(z, x)$ we still produce a result that demonstrates that in the resonant case $\lambda=\lambda_{1}$ a positive solution exists. Afterwards, dropping the assumption describing the generalized version of the Ambrosetti-Rabinowitz condition, we present another set of hypotheses on $j(z, x)$ which still guarantee the existence of a positive solution for problem (1.1) in the case of near resonance from the left at $\lambda_{1}$. Knowing that in general we should not expect having positive solutions to (1.1) in the case of near resonance from the right, i.e., $\lambda>\lambda_{1}$ close to $\lambda_{1}$, in this situation we supply existence and multiplicity results for nontrivial solutions.

Our results extend in a nonsmooth quasilinear framework several classical properties of single- or multi-valued semilinear Dirichlet boundary value problems at non-resonance, resonance and near resonance. They allow one to cover a larger area of applicability in various nonsmooth and nonconvex problems arising in mechanics and engineering (see [15], [17]). The main tools in our approach are minimax theorems, nonsmooth analysis, spectrum of the negative $p$-Laplacian, nonlinear regularity theory and nonlinear strong maximum principle. Examples illustrate all our results.

The rest of the paper is organized as follows. Section 2 deals with some mathematical preliminaries. Section 3 contains our results on positive solutions for non-resonance and resonance. Section 4 focuses on the positive solutions in the case of near resonance from the left. Section 5 is devoted to multiple solutions with near resonance from the right.

\section{MathematicAl BACKGround}

Our approach is variational based on the nonsmooth critical point theory which uses the subdifferential theory of locally Lipschitz functions. For easy reference, first we recall some basic definitions which we will need in the sequel. Let $X$ be a Banach space and $X^{*}$ its topological dual. By $\langle\cdot, \cdot\rangle$ we denote the duality bracket for the pair $\left(X, X^{*}\right)$. For a locally Lipschitz function $\varphi: X \rightarrow \mathbb{R}$, the generalized directional derivative $\varphi^{0}(x ; h)$ of $\varphi$ at $x \in X$ in the direction $h \in X$ is defined by

$$
\varphi^{0}(x ; h)=\limsup _{\substack{x^{\prime} \rightarrow x \\ \lambda \downarrow 0}} \frac{\varphi\left(x^{\prime}+\lambda h\right)-\varphi\left(x^{\prime}\right)}{\lambda},
$$

whereas the generalized gradient $\partial \varphi(x)$ of $\varphi$ at $x \in X$ is introduced as

$$
\partial \varphi(x)=\left\{x^{*} \in X^{*}:\left\langle x^{*}, h\right\rangle \leq \varphi^{0}(x ; h), \forall h \in X\right\}
$$

(see Clarke [5]). We say that $x \in X$ is a critical point of $\varphi$ if $0 \in \partial \varphi(x)$. A locally Lipschitz function $\varphi: X \rightarrow \mathbb{R}$ satisfies the nonsmooth Palais-Smale condition at level $c \in \mathbb{R}$ (nonsmooth $\mathrm{PS}_{c}$-condition for short) if every sequence $\left\{x_{n}\right\} \subset X$ such that $\varphi\left(x_{n}\right) \rightarrow c$ and $m\left(x_{n}\right)=\inf \left\{\left\|x^{*}\right\|: x^{*} \in \partial \varphi\left(x_{n}\right)\right\} \rightarrow 0$ as $n \rightarrow \infty$ 
has a strongly convergent subsequence. The locally Lipschitz function $\varphi: X \rightarrow \mathbb{R}$ satisfies the nonsmooth Palais-Smale condition (nonsmooth PS-condition for short) if it satisfies the nonsmooth $\mathrm{PS}_{c}$-condition for every $c \in \mathbb{R}$. It is also said that a locally Lipschitz function $\varphi: X \rightarrow \mathbb{R}$ satisfies the nonsmooth Cerami condition at level $c \in \mathbb{R}$ (nonsmooth $\mathrm{C}_{c}$-condition for short) if every sequence $\left\{x_{n}\right\} \subset X$ such that $\varphi\left(x_{n}\right) \rightarrow c$ and $\left(1+\left\|x_{n}\right\|\right) m\left(x_{n}\right) \rightarrow 0$ as $n \rightarrow \infty$ has a strongly convergent subsequence.

In this paper we use two minimax principles in the nonsmooth critical point theory. The first one is the nonsmooth version of the classical mountain pass theorem (see [4], [8], [15]).

Theorem 2.1. Let $X$ be a reflexive Banach space and let $\varphi: X \rightarrow \mathbb{R}$ be a locally Lipschitz function. Suppose that for some $\rho>0$ and $x_{1}, x_{2} \in X$ with $\left\|x_{1}-x_{2}\right\|>\rho$ one has

$$
\max \left\{\varphi\left(x_{1}\right), \varphi\left(x_{2}\right)\right\}<\beta:=\inf \left\{\varphi(x):\left\|x-x_{1}\right\|=\rho\right\} .
$$

If the function $\varphi$ satisfies the nonsmooth $\mathrm{C}_{c}$-condition (in particular, the nonsmooth $\mathrm{PS}_{c}$-condition), with

$$
c=\inf _{\gamma \in \Gamma} \max _{t \in[0,1]} \varphi(\gamma(t))
$$

where

$$
\Gamma=\left\{\gamma \in C([0,1], X): \gamma(0)=x_{1}, \gamma(1)=x_{2}\right\},
$$

then $\varphi$ has a critical point $x_{0} \in X$ with $\varphi\left(x_{0}\right)=c \geq \beta$.

The second minimax principle that we need is the nonsmooth variant of the Brezis-Nirenberg theorem with local linking (see [12], [19]).

Theorem 2.2. If $X$ is a reflexive Banach space admitting a direct sum decomposition $X=Y \oplus V$ with $\operatorname{dim} Y<+\infty, \varphi: X \rightarrow \mathbb{R}$ is a locally Lipschitz function which is bounded below, satisfies the nonsmooth PS-condition, $\varphi(0)=0, \inf _{X} \varphi<0$ and there exists $\rho>0$ such that

$$
\begin{cases}\varphi(y) \leq 0 & \text { if } y \in Y,\|y\| \leq \rho \\ \varphi(v) \geq 0 & \text { if } v \in V,\|v\| \leq \rho\end{cases}
$$

then $\varphi$ has at least two nontrivial critical points.

Finally we recall some facts about the spectrum of the negative $p$-Laplacian with Dirichlet boundary condition. Considering the nonlinear eigenvalue problem

$$
\left\{\begin{array}{l}
-\operatorname{div}\left(\|D x(z)\|^{p-2} D x(z)\right)=\lambda|x(z)|^{p-2} x(z) \text { a.e. on } Z, \\
\left.x\right|_{\partial Z}=0, \lambda \in \mathbb{R},
\end{array}\right.
$$

the least real number $\lambda$, denoted $\lambda_{1}$, for which problem (2.1) has a nontrivial solution in $W_{0}^{1, p}(Z)$, is called the principal eigenvalue of $\left(-\Delta_{p}, W_{0}^{1, p}(Z)\right)$. We know that $\lambda_{1}$ is positive, isolated and simple. There is the following variational characterization of $\lambda_{1}>0$ using Rayleigh quotient:

$$
\lambda_{1}=\inf \left\{\frac{\|D x\|_{p}^{p}}{\|x\|_{p}^{p}}: x \in W_{0}^{1, p}(Z), x \neq 0\right\} .
$$

This infimum is actually realized at the normalized eigenfunction $u_{1}$. We have that $u_{1} \in C^{1, \beta}(\bar{Z})$ with $0<\beta<1$ and we may assume $u_{1}(z)>0$ for all $z \in Z$ (see, e.g., $\left[8\right.$, p. 117]). Throughout the rest of the paper we keep the notation for $u_{1}$. 


\section{Positive solutions with non-Resonance And Resonance}

First, we establish the existence of positive smooth solutions for problem (1.1) in the non-resonant case. The hypotheses on the nonsmooth potential $j(z, x)$ are the following:

$\mathrm{H}(j)_{1} \quad j: Z \times \mathbb{R} \rightarrow \mathbb{R}$ is a function such that $j(z, 0)=0$ a.e. on $Z$ and

(i) for all $x \in \mathbb{R}, z \mapsto j(z, x)$ is measurable;

(ii) for almost all $z \in Z, x \mapsto j(z, x)$ is locally Lipschitz;

(iii) for almost all $z \in Z$, all $x \in \mathbb{R}$ and all $u \in \partial j(z, x)$, we have

$$
|u| \leq a(z)+c|x|^{r-1} \text { with } a \in L^{\infty}(Z)_{+}, c>0, p<r<p^{*}
$$

where $p^{*}=\left\{\begin{array}{cc}\frac{N p}{N-p} & \text { if } N>p \\ +\infty & \text { if } N \leq p\end{array}\right.$

(iv) there exist constants $\mu>p$ and $M>0$ such that

$$
\inf _{z \in Z} j(z, M)>0 \text { and } \mu j(z, x) \leq-j^{0}(z, x ;-x) \text { a.a. } z \in Z \text {, all } x \geq M \text {; }
$$

(v) $\limsup _{x \rightarrow 0^{+}} \frac{j(z, x)}{x^{p}} \leq 0$ uniformly for almost all $z \in Z$;

(vi) for almost all $z \in Z$, all $x>0$ and all $u \in \partial j(z, x)$, we have $u \geq-\xi x^{p-1}$ with a constant $\xi \geq 0$.

Assumption $\mathrm{H}(j)_{1}$ (iv) is a nonsmooth variant of the Ambrosetti-Rabinowitz condition in [1]. Notice that here we do not require the sign condition $j(z, x)>0$ for almost all $z \in Z$ and all $|x| \geq M$.

Theorem 3.1. If hypotheses $\mathrm{H}(j)_{1}$ hold and $\lambda<\lambda_{1}$, then problem (1.1) has a solution $x \in C_{0}^{1}(\bar{Z})$ with $x(z)>0$ for all $z \in Z$.

Proof. Let $\tau: \mathbb{R} \rightarrow \mathbb{R}$ be the Lipschitz continuous truncation function

$$
\tau(x)= \begin{cases}x & \text { if } x \geq 0 \\ 0 & \text { if } x<0 .\end{cases}
$$

Let $j_{1}(z, x)=j(z, \tau(x))$. For almost all $z \in Z, x \mapsto j_{1}(z, x)$ is locally Lipschitz and $j_{1}(z, \cdot)$ has the generalized gradient

$$
\partial j_{1}(z, x)=\left\{\begin{array}{ll}
\{0\} & \text { if } x<0, \\
\partial j(z, x) & \text { if } x>0
\end{array} \text { and } \partial j_{1}(z, 0) \subset \operatorname{conv}\{\{0\} \cup \partial j(z, 0)\} .\right.
$$

We consider the functional $\varphi_{1, \lambda}: W_{0}^{1, p}(Z) \rightarrow \mathbb{R}$ defined by

$$
\varphi_{1, \lambda}(x)=\frac{1}{p}\|D x\|_{p}^{p}-\frac{\lambda}{p}\|x\|_{p}^{p}-\int_{Z} j_{1}(z, x(z)) d z, \quad \forall x \in W_{0}^{1, p}(Z) .
$$

By $\mathrm{H}(j)_{1}(\mathrm{i})-(\mathrm{iii}), \varphi_{1, \lambda}$ is Lipschitz continuous on bounded sets, so locally Lipschitz.

Claim 1. $\varphi_{1, \lambda}$ satisfies the nonsmooth PS-condition.

Let $\left\{x_{n}\right\} \subset W_{0}^{1, p}(Z)$ be such that $\left|\varphi_{1, \lambda}\left(x_{n}\right)\right| \leq M_{1}$ for some constant $M_{1}>0$, all $n \geq 1$ and $m\left(x_{n}\right) \rightarrow 0$ as $n \rightarrow \infty$. Since the set

$$
\partial \varphi_{1, \lambda}\left(x_{n}\right) \subset W^{-1, p^{\prime}}(Z)=W_{0}^{1, p}(Z)^{*} \quad\left(1 / p+1 / p^{\prime}=1\right)
$$

is w-compact and the norm functional on a Banach space is weakly lower semicontinuous, we can find $x_{n}^{*} \in \partial \varphi_{1, \lambda}\left(x_{n}\right)$ such that $m\left(x_{n}\right)=\left\|x_{n}^{*}\right\|, n \geq 1$. 
Let $A: W_{0}^{1, p}(Z) \rightarrow W^{-1, p^{\prime}}(Z)$ be the negative $p$-Laplacian that is the nonlinear operator defined by

$$
\langle A(x), y\rangle=\int_{Z}\|D x(z)\|^{p-2}(D x(z), D y(z))_{\mathbb{R}^{N}} d z .
$$

It is known that $A$ is monotone and demicontinuous, hence maximal monotone. For every $n \geq 1$ we have

$$
x_{n}^{*}=A\left(x_{n}\right)-\lambda\left|x_{n}\right|^{p-2} x_{n}-u_{n}
$$

with $u_{n} \in L^{r^{\prime}}(Z)$, where $1 / r+1 / r^{\prime}=1$, satisfying $u_{n}(z) \in \partial j_{1}\left(z, x_{n}(z)\right)$ a.e. on $Z$. The choice of the sequence $\left\{x_{n}\right\} \subset W_{0}^{1, p}(Z)$ ensures

$$
\left|\left\langle x_{n}^{*}, x_{n}\right\rangle\right| \leq \varepsilon_{n}\left\|x_{n}\right\| \text { with } \varepsilon_{n} \downarrow 0,
$$

which implies that

$$
-\left\|D x_{n}\right\|_{p}^{p}+\lambda\left\|x_{n}\right\|_{p}^{p}-\int_{Z} j_{1}^{0}\left(z, x_{n}(z) ;-x_{n}(z)\right) d z \leq \varepsilon_{n}\left\|x_{n}\right\| .
$$

We also have

$$
\frac{1}{p}\left\|D x_{n}\right\|_{p}^{p}-\frac{\lambda}{p}\left\|x_{n}\right\|_{p}^{p}-\int_{Z} j_{1}\left(z, x_{n}(z)\right) d z \leq M_{1} .
$$

Combining (3.4) and (3.5), we obtain

$$
\begin{aligned}
& \left(\frac{\mu}{p}-1\right)\left\|D x_{n}\right\|_{p}^{p}-\lambda\left(\frac{\mu}{p}-1\right)\left\|x_{n}\right\|_{p}^{p} \\
& -\int_{Z}\left[\mu j_{1}\left(z, x_{n}(z)\right)+j_{1}^{0}\left(z, x_{n}(z) ;-x_{n}(z)\right)\right] d z \leq \mu M_{1}+\varepsilon_{n}\left\|x_{n}\right\| .
\end{aligned}
$$

Note that (3.2) yields

$$
j_{1}\left(z, x_{n}(z)\right)=0 \text { and } j_{1}^{0}\left(z, x_{n}(z) ;-x_{n}(z)\right)=0 \text { a.e. on }\left\{x_{n} \leq 0\right\} .
$$

So, by hypotheses $\mathrm{H}(j)_{1}$ (iii) and (iv), we have

$$
\begin{aligned}
& -\int_{Z}\left[\mu j_{1}\left(z, x_{n}(z)\right)+j_{1}^{0}\left(z, x_{n}(z) ;-x_{n}(z)\right)\right] d z \\
& =-\int_{\left\{0<x_{n}<M\right\}}\left[\mu j\left(z, x_{n}(z)\right)+j^{0}\left(z, x_{n}(z) ;-x_{n}(z)\right)\right] d z \\
& -\int_{\left\{x_{n} \geq M\right\}}\left[\mu j\left(z, x_{n}(z)\right)+j^{0}\left(z, x_{n}(z) ;-x_{n}(z)\right)\right] d z \geq-\beta_{1}, \quad \forall n \geq 1,
\end{aligned}
$$

for some constant $\beta_{1}>0$. Returning to (3.6) and using (3.7), we derive

$$
\left(\frac{\mu}{p}-1\right)\left\|D x_{n}\right\|_{p}^{p}-\lambda\left(\frac{\mu}{p}-1\right)\left\|x_{n}\right\|_{p}^{p} \leq \mu M_{1}+\varepsilon_{n}\left\|x_{n}\right\|+\beta_{1} .
$$

In order to show the boundedness of $\left\{x_{n}\right\}$ in $W_{0}^{1, p}(Z)$, we may suppose without loss of generality that $\lambda>0$. Then by $(2.2)$ it follows that

$$
\left(\frac{\mu}{p}-1\right)\left(1-\frac{\lambda}{\lambda_{1}}\right)\left\|D x_{n}\right\|_{p}^{p} \leq \mu M_{1}+\varepsilon_{n}\left\|x_{n}\right\|+\beta_{1}, \quad \forall n \geq 1 .
$$

Because $\lambda<\lambda_{1}$ and $\mu>p>1$, from (3.8) we infer that $\left\{x_{n}\right\} \subset W_{0}^{1, p}(Z)$ is bounded. So by passing to a subsequence if necessary, we may assume

$$
x_{n} \stackrel{\mathrm{w}}{\rightarrow} x \text { in } W_{0}^{1, p}(Z), x_{n} \rightarrow x \text { in } L^{r}(Z), x_{n}(z) \rightarrow x(z) \text { a.e. on } Z
$$


and $\left|x_{n}(z)\right| \leq k(z)$ a.e. on $Z$, for all $n \geq 1$, with $k \in L^{r}(Z)$. The inequality $\left|\left\langle x_{n}^{*}, x_{n}-x\right\rangle\right| \leq \varepsilon_{n}\left\|x_{n}-x\right\|$ reads

$$
\left.\left|\left\langle A\left(x_{n}\right), x_{n}-x\right\rangle-\lambda \int_{Z}\right| x_{n}\right|^{p-2} x_{n}\left(x_{n}-x\right) d z-\int_{Z} u_{n}\left(x_{n}-x\right) d z \mid \leq \varepsilon_{n}\left\|x_{n}-x\right\| .
$$

Since

$$
\lambda \int_{Z}\left|x_{n}\right|^{p-2} x_{n}\left(x_{n}-x\right) d z \rightarrow 0 \text { and } \int_{Z} u_{n}\left(x_{n}-x\right) d z \rightarrow 0 \text { as } n \rightarrow \infty,
$$

it results that $\lim _{n \rightarrow \infty}\left\langle A\left(x_{n}\right), x_{n}-x\right\rangle=0$. The operator $A$ being maximal monotone, it is generalized pseudomonotone and so $\left\langle A\left(x_{n}\right), x_{n}\right\rangle \rightarrow\langle A(x), x\rangle$, or equivalently, $\left\|D x_{n}\right\|_{p} \rightarrow\|D x\|_{p}$. Recalling that $D x_{n} \stackrel{\text { w }}{\rightarrow} D x$ in $L^{p}\left(Z, \mathbb{R}^{N}\right)$ and $L^{p}\left(Z, \mathbb{R}^{N}\right)$ being uniformly convex we have $D x_{n} \rightarrow D x$ in $L^{p}\left(Z, \mathbb{R}^{N}\right)$, which means $x_{n} \rightarrow x$ in $W_{0}^{1, p}(Z)$. This proves Claim 1 .

Claim 2. There exists $\rho>0$ such that $\beta:=\inf \left\{\varphi_{1, \lambda}(x):\|x\|=\rho\right\}>0$. that

By hypothesis $\mathrm{H}(j)_{1}(\mathrm{v})$, given $\varepsilon>0$, we can find a number $\delta=\delta(\varepsilon)>0$ such

$$
j_{1}(z, x)=j(z, x) \leq \frac{\varepsilon}{p} x^{p} \text { for a.a. } z \in Z, \forall x \in[0, \delta] .
$$

On the other hand, due to hypothesis $\mathrm{H}(j)_{1}$ (iii) and the mean value theorem for locally Lipschitz functions we can find a number $c_{\varepsilon}>0$ such that

$$
j_{1}(z, x)=j(z, x) \leq c_{\varepsilon} x^{r} \text { for a.a. } z \in Z, \forall x \geq \delta .
$$

From (3.9) and (3.10) we see that

$$
j_{1}(z, x) \leq \frac{\varepsilon}{p}|x|^{p}+c_{\varepsilon}|x|^{r} \text { for a.a. } z \in Z, \forall x \in \mathbb{R} .
$$

It is clear that for obtaining the estimate in Claim 2 it suffices to admit $\lambda>0$. Therefore, by (3.3), (3.11) and (2.2), we have

$$
\varphi_{1, \lambda}(x) \geq \frac{1}{p}\left(1-\frac{\lambda+\varepsilon}{\lambda_{1}}\right)\|D x\|_{p}^{p}-c_{1}\|D x\|_{p}^{r}, \quad \forall x \in W_{0}^{1, p}(Z),
$$

for some constant $c_{1}=c_{1}(\varepsilon)>0$. Because $\lambda<\lambda_{1}$, choosing $\varepsilon>0$ such that $\lambda+\varepsilon<\lambda_{1}$ and taking into account that $p<r$ and $\|D \cdot\|_{p}$ is an equivalent norm on $W_{0}^{1, p}(Z)$, from (3.12) we see that Claim 2 is satisfied for $\rho>0$ sufficiently small.

Claim 3. There exists $\hat{x} \in W_{0}^{1, p}(Z)$ with $\|\hat{x}\|>\rho$ such that $\varphi_{1, \lambda}(\hat{x})<\varphi_{1, \lambda}(0)=0$.

For almost all $z \in Z$ and all $x \in \mathbb{R}$, the function $s \mapsto \frac{1}{s^{\mu}} j(z, s x)$ is locally Lipschitz on $(0,+\infty)$ and we have

$$
\partial_{s}\left(\frac{1}{s^{\mu}} j(z, s x)\right) \subseteq-\frac{\mu}{s^{\mu+1}} j(z, s x)+\frac{1}{s^{\mu}} \partial_{x} j(z, s x) x .
$$

Here by $\partial_{s}$ and $\partial_{x}$ we denote the generalized gradient with respect to $s>0$ and $x \in \mathbb{R}$, respectively. Using the mean value theorem for locally Lipschitz functions, for $s>1$ we can find $\theta \in(1, s)$ such that

$$
\begin{gathered}
\frac{1}{s^{\mu}} j(z, s x)-j(z, x) \in\left(-\frac{\mu}{\theta^{\mu+1}} j(z, \theta x)+\frac{1}{\theta^{\mu}} \partial_{x} j(z, \theta x) x\right)(s-1) \\
=\frac{s-1}{\theta^{\mu+1}}\left(-\mu j(z, \theta x)+\partial_{x} j(z, \theta x) \theta x\right) .
\end{gathered}
$$


By hypothesis $\mathrm{H}(j)_{1}$ (iv), for almost all $z \in Z$ and all $x \geq M$, this implies

$$
\frac{1}{s^{\mu}} j_{1}(z, s x)-j_{1}(z, x) \geq \frac{s-1}{\theta^{\mu+1}}\left(-\mu j(z, \theta x)-j^{0}(z, \theta x ;-\theta x)\right) \geq 0 .
$$

Then for almost all $z \in Z$ and all $x \geq M$ we have

$$
j_{1}(z, x)=j_{1}\left(z, \frac{x}{M} M\right) \geq\left(\frac{x}{M}\right)^{\mu} j(z, M) \geq\left(\frac{x}{M}\right)^{\mu} \inf _{z \in Z} j(z, M) .
$$

From this inequality and the first part of hypothesis $\mathrm{H}(j)_{1}$ (iv), it is seen that given $\eta>0$, we can find $M_{\eta}>0$ such that

$$
j_{1}(z, x) \geq \frac{\eta}{p} x^{p} \text { for a.a. } z \in Z, \forall x \geq M_{\eta} .
$$

Combining (3.13) and $\mathrm{H}(j)_{1}(\mathrm{iii})$ shows that for a constant $c_{\eta}>0$ one has

$$
j_{1}(z, x) \geq \frac{\eta}{p} x^{p}-c_{\eta} \text { for a.a. } z \in Z, \forall x \geq 0 .
$$

By (3.3), (2.2), (3.14) it turns out that

$$
\varphi_{1, \lambda}\left(t u_{1}\right) \leq \frac{t^{p}}{p}\left(1-\frac{\lambda}{\lambda_{1}}-\frac{\eta}{\lambda_{1}}\right)\left\|D u_{1}\right\|_{p}^{p}+\beta_{3}, \forall t>0
$$

with a constant $\beta_{3}>0$. Now choose $\eta>\lambda_{1}-\lambda>0$. Claim 3 follows because $\varphi_{1, \lambda}\left(t u_{1}\right) \rightarrow-\infty$ as $t \rightarrow+\infty$.

Claims 1, 2 and 3 permit the use of Theorem 2.1. We obtain $x \in W_{0}^{1, p}(Z)$ such that $\varphi_{1, \lambda}(x) \geq \beta>0=\varphi_{1, \lambda}(0)$, thereby $x \neq 0$, and $0 \in \partial \varphi_{1, \lambda}(x)$. The last inclusion ensures

$$
A(x)-\lambda|x|^{p-2} x=u \text { with } u \in L^{r^{\prime}}(Z), u(z) \in \partial j_{1}(z, x(z)) \text { a.e. on } Z \text {. }
$$

From nonlinear regularity theory (see, e.g., [8, p. 115]) we deduce $x \in C_{0}^{1}(\bar{Z})$.

Let $x^{-}=\max \{-x, 0\} \in W_{0}^{1, p}(Z)$ and suppose $x^{-} \neq 0$. Substituting

$$
D x^{-}(z)= \begin{cases}-D x(z) & \text { a.e. on }\{x<0\} \\ 0 & \text { a.e. on }\{x \geq 0\}\end{cases}
$$

in $\left\langle A(x),-x^{-}\right\rangle-\lambda\left\langle|x|^{p-2} x,-x^{-}\right\rangle=\left\langle u,-x^{-}\right\rangle$(see (3.15)), we obtain on the basis of $(3.2)$ that

$$
\left\|D x^{-}\right\|_{p}^{p}-\lambda\left\|x^{-}\right\|_{p}^{p}=-\int_{Z} u x^{-} d z=0 .
$$

Recalling $\lambda<\lambda_{1}$ and $x^{-} \neq 0$, this implies that $\left\|D x^{-}\right\|_{p}^{p}<\lambda_{1}\left\|x^{-}\right\|_{p}^{p}$, which contradicts $(2.2)$. Hence $x^{-}=0$ and so $x \geq 0, x \not \equiv 0$. Then by $\mathrm{H}(j)_{1}$ (vi), we have $A(x)-\lambda x^{p-1}=u \geq-\xi x^{p-1}$ a.e. on $\{x>0\}$, thus

$$
\operatorname{div}\left(\|D x(z)\|^{p-2} D x(z)\right) \leq(|\lambda|+\xi) x(z)^{p-1} \text { a.e. on } Z \text {. }
$$

Through the nonlinear strong maximum principle due to Vázquez [18] (see also Gasiński-Papageorgiou [8, p. 116]) applied with the function therein $\beta(u)=$ $(|\lambda|+\xi) u^{p-1}$ for $u \geq 0$, we conclude that $x(z)>0$ for all $z \in Z$. In view of (3.15) and (3.2) we note that $x$ solves (1.1) which completes the proof.

Example 3.2. Consider problem (1.1) with the following potential (for simplicity we drop the $z$-dependence):

$$
j(x)=\max \left\{\frac{1}{\eta}|x|^{\eta}, \frac{1}{r}|x|^{r}-\frac{c}{p}|x|^{p}\right\},
$$


for $c \geq 0, p<\eta<p^{*}, p \leq r<p^{*}$ and $c \geq 1$ if $p=r$. It is readily seen that hypothesis $\mathrm{H}(j)_{1}$ is fulfilled, so Theorem 3.1 applies to the corresponding problem (1.1).

If $p=2$, by strengthening assumption $\mathrm{H}(j)_{1}$ we obtain a converse result. Namely, we state

$\mathrm{H}(j)_{2} \quad j: Z \times \mathbb{R} \rightarrow \mathbb{R}$ is a function such that $j(z, 0)=0$ a.e. on $Z$, it satisfies $\mathrm{H}(j)_{1}(\mathrm{i})-(\mathrm{v})$ with $p=2$, and

(vi) ${ }^{\prime}$ for almost all $z \in Z$, all $x>0$ and all $u \in \partial j(z, x)$, one has $u>0$.

Theorem 3.3. If hypotheses $\mathrm{H}(j)_{2}$ hold, then problem (1.1) has a solution $x \in$ $C_{0}^{1}(\bar{Z})$ with $x(z)>0$ for all $z \in Z$ if and only if $\lambda<\lambda_{1}$.

Proof. The sufficiency part follows from Theorem 3.1 because assumption $\mathrm{H}(j)_{2}$ is stronger than $\mathrm{H}(j)_{1}$. For the necessity part let $x \in C_{0}^{1}(\bar{Z})$ be a positive solution of (1.1), thus

$$
-\Delta x(z)-\lambda x(z)=u(z) \text { a.e. on } Z
$$

with $u \in L^{r^{\prime}}(Z), u(z) \in \partial j(z, x(z))$ a.e. on $Z$. It yields

$$
-\int_{Z} \Delta x(z) u_{1}(z) d z-\lambda \int_{Z} x(z) u_{1}(z) d z=\int_{Z} u(z) u_{1}(z) d z>0
$$

according to hypothesis $\mathrm{H}(j)_{2}(\mathrm{vi})^{\prime}$. This implies

$$
-\int_{Z} x(z) \Delta u_{1}(z) d z=\lambda_{1} \int_{Z} x(z) u_{1}(z) d z>\lambda \int_{Z} x(z) u_{1}(z) d z,
$$

which is equivalent to $\lambda_{1}>\lambda$.

In the following we focus on the resonant case $\lambda=\lambda_{1}$. To this end we need an auxiliary result.

Lemma 3.4. If $\vartheta_{1} \in L^{\infty}(Z)_{+}, \vartheta_{1}(z) \leq \lambda_{1}$ a.e. on $Z$ with strict inequality on $a$ set of positive measure, then there exists a constant $\xi_{0}>0$ such that

$$
\psi(x):=\|D x\|_{p}^{p}-\int_{Z} \vartheta_{1}(z)|x(z)|^{p} d z \geq \xi_{0}\|D x\|_{p}^{p}, \quad \forall x \in W_{0}^{1, p}(Z) .
$$

Proof. From (2.2) and the hypothesis on $\vartheta_{1}$, we see that $\psi \geq 0$. Suppose that the conclusion of the lemma is not true. Exploiting the $p$-homogeneity of $\psi$, we find $\left\{x_{n}\right\} \subset W_{0}^{1, p}(Z)$ such that

$$
\psi\left(x_{n}\right) \downarrow 0 \text { as } n \rightarrow \infty \text { and }\left\|D x_{n}\right\|_{p}=1 \text { for all } n \geq 1 \text {. }
$$

By passing to a subsequence if necessary, we may assume that

$$
x_{n} \stackrel{\mathrm{w}}{\rightarrow} x \text { in } W_{0}^{1, p}(Z), x_{n} \rightarrow x \text { in } L^{p}(Z), x_{n}(z) \rightarrow x(z) \text { a.e. on } Z
$$

and $\left|x_{n}(z)\right| \leq k(z)$ a.e. on $Z$, for $k \in L^{p}(Z)$. Since the norm on a Banach space is weakly lower semicontinuous, in the limit as $n \rightarrow \infty$ we obtain

$$
\|D x\|_{p}^{p} \leq \int_{Z} \vartheta_{1}(z)|x(z)|^{p} d z \leq \lambda_{1}\|x\|_{p}^{p} .
$$

By (2.2), it follows that $x=t u_{1}, t \in \mathbb{R}$. If $x=0$, it turns out that $\left\|D x_{n}\right\|_{p} \rightarrow 0$ because $\psi\left(x_{n}\right) \rightarrow 0$, which contradicts $\left\|D x_{n}\right\|_{p}=1$. Thus $x=t u_{1}, t \neq 0$. From (3.16) we have $\|D x\|_{p}^{p}<\lambda_{1}\|x\|_{p}^{p}$ contradicting (2.2). 
Restricting $\mathrm{H}(j)_{1}$ (with $1<p<+\infty$ ) in a different way than was done in $\mathrm{H}(j)_{2}$ for $p=2$, in particular allowing the elements of $\partial j(z, x)$ to be not everywhere positive whenever $x>0$, we incorporate in our considerations resonant problems, that is, for $\lambda=\lambda_{1}$. We formulate the new hypotheses:

$\mathrm{H}(j)_{3} \quad j: Z \times \mathbb{R} \rightarrow \mathbb{R}$ is a function such that $j(z, 0)=0$ a.e. on $Z$, it satisfies $\mathrm{H}(j)_{1}(\mathrm{i})-(\mathrm{iv}),(\mathrm{vi})$, and

$(\mathrm{v})^{\prime}$ there exists $\vartheta \in L^{\infty}(Z)$ such that $\vartheta(z) \leq 0$ a.e. on $Z$ with strict inequality on a set of positive measure and

$$
\limsup _{x \rightarrow 0^{+}} \frac{p j(z, x)}{x^{p}} \leq \vartheta(z) \text { uniformly for almost all } z \in Z ;
$$

(vii) for almost all $z \in Z$, all $x, y>0$ and $u \in \partial j(z, x), v \in \partial j(z, y)$ one has

$$
\left(\frac{u}{x^{p-1}}-\frac{v}{y^{p-1}}\right)(x-y) \geq 0 .
$$

Hypothesis $\mathrm{H}(j)_{3}$ (vii) extends a condition used in [11] in the context of asymptotically linear problems.

In order to deal with the resonant case $\lambda=\lambda_{1}$ we consider the locally Lipschitz function $\varphi_{1}: W_{0}^{1, p}(Z) \rightarrow \mathbb{R}$ defined by

$$
\varphi_{1}(x)=\frac{1}{p}\|D x\|_{p}^{p}-\frac{\lambda_{1}}{p}\|x\|_{p}^{p}-\int_{Z} j_{1}(z, x(z)) d z, \quad \forall x \in W_{0}^{1, p}(Z) .
$$

Here enters the modified potential function $j_{1}(z, x)=j(z, \tau(x))$ with the truncation $\tau: \mathbb{R} \rightarrow \mathbb{R}$ in (3.1).

The following auxiliary result on $\varphi_{1}$ in (3.17) extends [11, Lemma 2.4].

Lemma 3.5. Under hypotheses $\mathrm{H}(j)_{3}$, any sequence $\left\{x_{n}\right\} \subset W_{0}^{1, p}(\Omega)$ such that $x_{n} \geq 0$ a.e. in $\Omega$ and for which there exists $x_{n}^{*} \in \partial \varphi_{1}\left(x_{n}\right)$ provided $\left\langle x_{n}^{*}, x_{n}\right\rangle \rightarrow 0$ as $n \rightarrow \infty$ contains a relabelled subsequence satisfying

$$
\varphi_{1}\left(t x_{n}\right) \leq \frac{1+t^{p}}{p n}+\varphi_{1}\left(x_{n}\right), \quad \forall t>0, \forall n \geq 1 .
$$

Proof. By passing to a subsequence if necessary, we may assume

$$
-\frac{1}{n} \leq\left\langle x_{n}^{*}, x_{n}\right\rangle=\left\|D x_{n}\right\|_{p}^{p}-\lambda_{1}\left\|x_{n}\right\|_{p}^{p}-\int_{Z} u_{n} x_{n} d z \leq \frac{1}{n}, \quad \forall n \geq 1
$$

with $u_{n} \in L^{r^{\prime}}(Z)$ satisfying $u_{n}(z) \in \partial j_{1}\left(z, x_{n}(z)\right)$ for a.a. $z \in Z$. Denoting by $|\cdot|_{1}$ the Lebesgue measure on $\mathbb{R}$, we know that $j_{1}(z, \cdot)$ is locally Lipschitz for all $z \in Z \backslash D_{0}$, where $\left|D_{0}\right|_{1}=0$. With fixed $z \in Z \backslash D_{0}$ and $n \geq 1$, we introduce

$$
\zeta(t)=\frac{t^{p}}{p} u_{n}(z) x_{n}(z)-j_{1}\left(z, t x_{n}(z)\right), \quad \forall t>0 .
$$

The function $\zeta$ is differentiable for almost all $t>0$, and from assumption $\mathrm{H}(j)_{3}$ (vii), for a.a. $t>0$ we have

$$
\zeta^{\prime}(t)=t^{p-1} x_{n}(z)\left(u_{n}(z)-\frac{\frac{d}{d s} j_{1}\left(z, t x_{n}(z)\right)}{t^{p-1}}\right) \quad \begin{cases}\leq 0 & \text { if } t \geq 1, \\ \geq 0 & \text { if } t \in(0,1]\end{cases}
$$

because $\frac{d}{d s} j_{1}\left(z, t x_{n}(z)\right) \in \partial j_{1}\left(z, t x_{n}(z)\right)$, where $\frac{d}{d s} j_{1}(z, s)$ stands for the derivative of $j_{1}(z, s)$ with respect to $s$. This implies that

$$
\zeta(t) \leq \zeta(1), \quad \forall t>0 .
$$


Then, by (3.17)-(3.19), for $t>0$ and $n \geq 1$ we derive

$$
\varphi_{1}\left(t x_{n}\right) \leq \frac{t^{p}}{p n}+\int_{Z}\left(\frac{1}{p} u_{n}(z) x_{n}(z)-j_{1}\left(z, x_{n}(z)\right)\right) d z .
$$

Also by (3.17) and (3.18) for every $n \geq 1$ we have

$$
\varphi_{1}\left(x_{n}\right) \geq-\frac{1}{p n}+\int_{Z}\left(\frac{1}{p} u_{n}(z) x_{n}(z)-j_{1}\left(z, x_{n}(z)\right)\right) d z .
$$

Using (3.21) and (3.20) we achieve the desired conclusion.

We can now handle the resonant case.

Theorem 3.6. If hypotheses $\mathrm{H}(j)_{3}$ hold and $\lambda=\lambda_{1}$, then problem (1.1) has a solution $x \in C_{0}^{1}(\bar{Z})$ with $x(z)>0$ for all $z \in Z$.

Proof. First we show that $\varphi_{1}$ in (3.17) satisfies the nonsmooth $\mathrm{C}_{c}$-condition for any number $c>0$. Consider a sequence $\left\{x_{n}\right\} \subset W_{0}^{1, p}(Z)$ such that

$$
\varphi_{1}\left(x_{n}\right) \rightarrow c \text { and }\left(1+\left\|x_{n}\right\|\right) m\left(x_{n}\right) \rightarrow 0 \text { as } n \rightarrow \infty \text {. }
$$

We f ind $x_{n}^{*} \in \partial \varphi_{1}\left(x_{n}\right)$ with $m\left(x_{n}\right)=\left\|x_{n}^{*}\right\|$ and

$$
x_{n}^{*}=A\left(x_{n}\right)-\lambda_{1}\left|x_{n}\right|^{p-2} x_{n}-u_{n}
$$

for $u_{n} \in L^{r^{\prime}}(Z), u_{n}(z) \in \partial j_{1}\left(z, x_{n}(z)\right)$ a.e. on $Z$. We infer

$$
\left(1+\left\|x_{n}\right\|\right)\left|\left\langle x_{n}^{*}, v\right\rangle\right| \leq \varepsilon_{n}\|v\|, \quad \forall v \in W_{0}^{1, p}(Z),
$$

where $\varepsilon_{n} \downarrow 0$. Writing $x_{n}=x_{n}^{+}-x_{n}^{-}$with $x_{n}^{+}=\max \left\{x_{n}, 0\right\}$ and $x_{n}^{-}=\max \left\{-x_{n}, 0\right\}$, we set $v=-x_{n}^{-} \in W_{0}^{1, p}(Z)$ to obtain via (3.2) that

$$
\left\|D x_{n}^{-}\right\|_{p}^{p}-\lambda_{1}\left\|x_{n}^{-}\right\|_{p}^{p} \leq \varepsilon_{n} \frac{\left\|x_{n}^{-}\right\|}{1+\left\|x_{n}\right\|}<\varepsilon_{n} .
$$

This ensures that

$$
\varphi_{1}\left(-x_{n}^{-}\right)=\frac{1}{p}\left\|D x_{n}^{-}\right\|_{p}^{p}-\frac{\lambda_{1}}{p}\left\|x_{n}^{-}\right\|_{p}^{p} \rightarrow 0 \text { as } n \rightarrow \infty .
$$

Since $\varphi_{1}\left(x_{n}\right)=\varphi_{1}\left(x_{n}^{+}\right)+\varphi_{1}\left(-x_{n}^{-}\right)$, we obtain

$$
\varphi_{1}\left(x_{n}^{+}\right) \rightarrow c \text { as } n \rightarrow \infty .
$$

Suppose that $\left\|x_{n}\right\| \rightarrow \infty$ along a relabelled subsequence. Having $\left\|x_{n}\right\|^{p}=$ $\left\|x_{n}^{-}\right\|^{p}+\left\|x_{n}^{+}\right\|^{p}$ and admitting without loss of generality that $\left\|x_{n}^{+}\right\|=\left\|D x_{n}^{+}\right\|_{p}$, first we assume that $\left\|x_{n}^{+}\right\| \rightarrow \infty$. Let

$$
t_{n}=\frac{(2 p c)^{\frac{1}{p}}}{\left\|x_{n}^{+}\right\|} \text {and } y_{n}=t_{n} x_{n}^{+} .
$$

We see that

$$
x_{n}^{*}=v_{n}^{*}-A\left(x_{n}^{-}\right)+\lambda_{1}\left(x_{n}^{-}\right)^{p-1},
$$

where, according to (3.2),

$$
v_{n}^{*}=A\left(x_{n}^{+}\right)-\lambda_{1}\left(x_{n}^{+}\right)^{p-1}-u_{n} \in \partial \varphi_{1}\left(x_{n}^{+}\right) .
$$

We deduce $\left\langle v_{n}^{*}, x_{n}^{+}\right\rangle=\left\langle x_{n}^{*}, x_{n}^{+}\right\rangle \rightarrow 0$ as $n \rightarrow \infty$, which enables us to apply Lemma 3.5 and (3.23) for obtaining along a relabelled subsequence that

$$
\varphi_{1}\left(y_{n}\right)=\varphi_{1}\left(t_{n} x_{n}^{+}\right) \leq \frac{1+t_{n}^{p}}{p n}+\varphi_{1}\left(x_{n}^{+}\right) \rightarrow c \text { as } n \rightarrow \infty .
$$


Eventually passing to a subsequence, we may suppose

$$
y_{n} \stackrel{\mathrm{w}}{\rightarrow} y \text { in } W_{0}^{1, p}(Z), y_{n} \rightarrow y \text { in } L^{r}(Z), y_{n}(z) \rightarrow y(z) \text { a.e. on } Z
$$

and $y_{n}(z) \leq k(z)$ a.e. on $Z$, for all $n \geq 1$, with $k \in L^{r}(Z)$. If $y=0$, then

$$
\varphi_{1}\left(y_{n}\right)=2 c-\frac{\lambda_{1}}{p}\left\|y_{n}\right\|_{p}^{p}-\int_{Z} j_{1}\left(z, y_{n}(z)\right) d z \rightarrow 2 c .
$$

Comparing (3.25) and (3.24), we achieve a contradiction since $c>0$. This proves that $y \neq 0$ and clearly $y \geq 0$. Thus the set $C=\{y>0\} \subset \mathbb{R}^{N}$ has the Lebesgue measure $|C|_{N}>0$. So $x_{n}^{+}(z) \rightarrow+\infty$ a.e. on $C$. On the other hand, (3.23) shows

$$
p \varphi_{1}\left(x_{n}^{+}\right)-\left\langle v_{n}^{*}, x_{n}^{+}\right\rangle \rightarrow p c \text { as } n \rightarrow \infty \text {. }
$$

As in Claim 3 of the proof of Theorem 3.1, using $\mathrm{H}(j)_{1}$ (iii)-(iv) we derive

$$
j_{1}(z, x) \geq a_{0} x^{\mu}-\hat{\beta} \text {, for a.a. } z \in Z, \forall x \geq 0,
$$

with constants $a_{0}>0$ and $\hat{\beta}>0$. Due to $\mathrm{H}(j)_{1}(\mathrm{iv})$, this gives

$$
u x-p j(z, x) \geq(\mu-p) j(z, x) \geq(\mu-p) a_{0} x^{\mu}-(\mu-p) \hat{\beta},
$$

for almost all $z \in Z$, all $x \geq M$ and all $u \in \partial j_{1}(z, x)$. We conclude that

$$
u x-p j_{1}(z, x) \rightarrow+\infty \text { as } x \rightarrow+\infty
$$

uniformly for a.a. $z \in Z$ and all $u \in \partial j_{1}(z, x)$. Thus we can find $\hat{\eta}>0$ such that

$$
u x-p j_{1}(z, x) \geq 1 \text { for a.a. } z \in Z, \forall x \geq \hat{\eta}, \forall u \in \partial j_{1}(z, x) .
$$

In addition, from $\mathrm{H}(j)_{1}$ (iii) there exists $\hat{\eta}_{1}>0$ satisfying

$$
\left|u x-p j_{1}(z, x)\right| \leq \hat{\eta}_{1} \text { for a.a. } z \in Z, \forall x \in[0, \hat{\eta}], \forall u \in \partial j_{1}(z, x) .
$$

Consequently, a constant $\eta_{2} \in \mathbb{R}$ can be found such that

$$
u x-p j_{1}(z, x) \geq \eta_{2} \text { for a.a. } z \in Z, \forall x \geq 0, \forall u \in \partial j_{1}(z, x) .
$$

Then from (3.28) and (3.2) we have

$$
\begin{aligned}
p \varphi_{1}\left(x_{n}^{+}\right)-\left\langle v_{n}^{*}, x_{n}^{+}\right\rangle & =\int_{Z}\left(u_{n}(z) x_{n}^{+}(z)-p j_{1}\left(z, x_{n}^{+}(z)\right)\right) d z \\
& \geq \int_{C}\left(u_{n}(z) x_{n}^{+}(z)-p j_{1}\left(z, x_{n}^{+}(z)\right)\right) d z+\eta_{2}|Z \backslash C|_{N} .
\end{aligned}
$$

By (3.27) and $x_{n}^{+}(z) \rightarrow+\infty$ a.e. on $C$ it follows that

$$
\int_{C}\left(u_{n}(z) x_{n}^{+}(z)-p j_{1}\left(z, x_{n}^{+}(z)\right)\right) d z \rightarrow+\infty .
$$

Since this contradicts (3.26), we infer $\left\{x_{n}^{+}\right\} \subset W_{0}^{1, p}(Z)$ is bounded. Therefore we must have that $\left\|x_{n}^{-}\right\| \rightarrow \infty$.

Set $\hat{y}_{n}=\frac{x_{n}}{\left\|x_{n}\right\|}$. We may assume

$$
\hat{y}_{n} \stackrel{\mathrm{w}}{\rightarrow} \hat{y} \text { in } W_{0}^{1, p}(Z), \hat{y}_{n} \rightarrow \hat{y} \text { in } L^{p}(Z), \hat{y}_{n}(z) \rightarrow \hat{y}(z) \text { a.e. on } Z
$$

and $\left|\hat{y}_{n}(z)\right| \leq \hat{k}(z)$ a.e. on $Z$, for all $n \geq 1$, with $\hat{k} \in L^{p}(Z)$. Because $\left\{x_{n}^{+}\right\} \subset$ $W_{0}^{1, p}(Z)$ is bounded, we have $\hat{y} \leq 0$. By (3.22) we obtain

$$
\left\|D \hat{y}_{n}^{-}\right\|_{p}^{p}-\lambda_{1}\left\|\hat{y}_{n}^{-}\right\|_{p}^{p}<\varepsilon_{n}
$$

provided $n$ is sufficiently large to have $\left\|x_{n}\right\| \geq 1$. Letting $n \rightarrow \infty$ gives $\|D \hat{y}\|_{p}^{p} \leq$ $\lambda_{1}\|\hat{y}\|_{p}^{p}$. Recalling that $\hat{y} \leq 0$, this implies $\hat{y}=-t u_{1}$ with $t \geq 0$. If $\hat{y}=0$, then from 
(3.29) we have $\hat{y}_{n} \rightarrow 0$ in $W_{0}^{1, p}(Z)$, which contradicts that $\left\|\hat{y}_{n}\right\|=1$. It remains that $\hat{y}=-t u_{1}$ with $t>0$, thus $x_{n}(z) \rightarrow-\infty$ a.e. on $Z$.

The choice of the sequence $\left\{x_{n}\right\} \subset W_{0}^{1, p}(Z)$ shows

$$
p \varphi_{1}\left(x_{n}\right)-\left\langle x_{n}^{*}, x_{n}\right\rangle=\int_{Z}\left(u_{n}(z) x_{n}(z)-p j_{1}\left(z, x_{n}(z)\right)\right) d z \rightarrow p c \text { as } n \rightarrow \infty .
$$

By (3.2) and the definition of the truncated potential $j_{1}(z, x)$, we obtain

$$
\int_{\left\{x_{n}<0\right\}}\left(u_{n}(z) x_{n}(z)-p j_{1}\left(z, x_{n}(z)\right)\right) d z=0, \quad \forall n \geq 1 .
$$

Since $\left\{x_{n}^{+}\right\} \subset W_{0}^{1, p}(Z)$ is bounded and $\left|\left\{x_{n} \geq 0\right\}\right|_{N} \rightarrow 0$ as $n \rightarrow \infty$ because $x_{n}(z) \rightarrow-\infty$ a.e. on $Z$, we have

$$
\int_{\left\{x_{n} \geq 0\right\}}\left(u_{n}(z) x_{n}(z)-p j_{1}\left(z, x_{n}(z)\right)\right) d z \rightarrow 0 \text { as } n \rightarrow \infty .
$$

So finally we get

$$
\int_{Z}\left(u_{n}(z) x_{n}(z)-p j_{1}\left(z, x_{n}(z)\right)\right) d z \rightarrow 0 \text { as } n \rightarrow \infty
$$

Comparing (3.30) and (3.31) a contradiction is reached because $c>0$. This proves the boundedness of $\left\{x_{n}\right\} \subset W_{0}^{1, p}(Z)$. From now on we can proceed as in Claim 1 of the proof of Theorem 3.1 to check that $\varphi_{1}$ satisfies the nonsmooth $\mathrm{C}_{c^{-}}$-condition for $c>0$.

From the hypotheses $\mathrm{H}(j)_{1}(\mathrm{iii}), \mathrm{H}(j)_{3}(\mathrm{v})^{\prime}$ and the mean value theorem for locally Lipschitz functions, given $\varepsilon>0$, there is $c_{\varepsilon}>0$ such that

$$
j_{1}(z, x) \leq \frac{1}{p}(\vartheta(z)+\varepsilon)|x|^{p}+c_{\varepsilon}|x|^{r} \text { for a.a. } z \in Z, \forall x \in \mathbb{R} .
$$

Consequently, for all $x \in W_{0}^{1, p}(Z)$, by (3.17) it is seen that

$$
\varphi_{1}(x) \geq \frac{1}{p}\|D x\|_{p}^{p}-\frac{1}{p} \int_{Z}\left(\lambda_{1}+\vartheta(z)\right)|x(z)|^{p} d z-\frac{\varepsilon}{p}\|x\|_{p}^{p}-c_{2}\|D x\|_{p}^{r},
$$

with a constant $c_{2}>0$. We set $\vartheta_{1}(z)=\vartheta(z)+\lambda_{1} \leq \lambda_{1}$ a.e. on $Z$ with strict inequality on a set of positive measure in view of $\mathrm{H}(j)_{3}(\mathrm{v})^{\prime}$. By Lemma 3.4 we find $\xi_{0}>0$ with the property

$$
\varphi_{1}(x) \geq \frac{1}{p}\left(\xi_{0}-\frac{\varepsilon}{\lambda_{1}}\right)\|D x\|_{p}^{p}-c_{2}\|D x\|_{p}^{r}, \quad \forall x \in W_{0}^{1, p}(Z) .
$$

Choosing $\varepsilon<\xi_{0} \lambda_{1}$, because $r>p$ there is $\rho>0$ satisfying

$$
\inf \left\{\varphi_{1}(x):\|x\|=\rho\right\}=\beta>0 .
$$

Furthermore, proceeding as for Claim 3 in the proof of Theorem 3.1 we achieve the assertion therein. Then using the inequality

$$
\inf _{\gamma \in \Gamma} \max _{t \in[0,1]} \varphi_{1}(\gamma(t)) \geq \beta>0,
$$

with $\Gamma$ as in Theorem 2.1, it follows that the nonsmooth $\mathrm{C}_{c}$-condition suffices for $c>0$ to be applied. The rest of the proof proceeds as that of Theorem 3.1. We obtain $x^{-}=0$ since if $x^{-}=t u_{1}$ with $t>0$, then $x=-t u_{1}$ and $\varphi_{1}(x)=0$, a contradiction. 
Example 3.7. Consider problem (1.1) at resonance $\lambda=\lambda_{1}$ with the locally Lipschitz potential given below:

$$
j(z, x)= \begin{cases}\frac{\vartheta(z)}{p}|x|^{p} & \text { if } x \leq 1, \\ \frac{1}{\mu} x^{\mu}+\frac{\vartheta(z)}{p} x^{p}-\frac{1}{\mu} & \text { if } x \geq 1,\end{cases}
$$

for $p<\mu<p^{*}$ and $\vartheta \in L^{\infty}(Z)$ where $\vartheta(z) \leq 0$ a.e. on $Z$ with strict inequality on a set of positive measure. It is straightforward to verify that assumptions $\mathrm{H}(j)_{3}$ are fulfilled, so Theorem 3.6 can be applied yielding a positive solution to problem (1.1) in the resonant case $\lambda=\lambda_{1}$ with $j(z, x)$ as above.

\section{Non-Negative Solutions With NEAR RESONANCE From the LEFT}

In this section the parameter $\lambda$ approaches $\lambda_{1}>0$ from the left. Our hypotheses on the potential $j(z, x)$ are the following:

$\mathrm{H}(j)_{4} j: Z \times \mathbb{R} \rightarrow \mathbb{R}$ is a function such that $j(z, 0)=0$ a.e. on $Z$, it satisfies $\mathrm{H}(j)_{1}(\mathrm{i})$, (ii), (v), (vi), and

(iii) $^{\prime}$ for almost all $z \in Z$, all $x \in \mathbb{R}$ and all $u \in \partial j(z, x)$, we have

$$
|u| \leq a(z)+c|x|^{p-1} \text { with } a \in L^{\infty}(Z)_{+}, c>0 ;
$$

(iv) ${ }^{\prime}$ there exists a constant $\varepsilon_{0}>0$ such that

$$
\liminf _{x \rightarrow+\infty} \frac{u}{x^{p-1}} \geq \varepsilon_{0} \quad \text { uniformly for almost all } z \in Z \text { and all } u \in \partial j(z, x) .
$$

Theorem 4.1. If hypotheses $\mathrm{H}(j)_{4}$ hold, then for all $\lambda \in\left(\lambda_{1}-\varepsilon_{0}, \lambda_{1}\right)$ problem (1.1) has a solution $x \in C_{0}^{1}(\bar{Z})$ with $x(z)>0$ for all $z \in Z$.

Proof. We again consider the locally Lipschitz functional $\varphi_{1, \lambda}: W_{0}^{1, p}(Z) \rightarrow \mathbb{R}$ defined by (3.3).

Claim 1. For every $\lambda \in\left(\lambda_{1}-\varepsilon_{0}, \lambda_{1}\right), \varphi_{1, \lambda}$ satisfies the nonsmooth PS-condition.

Suppose that $\left\{x_{n}\right\} \subset W_{0}^{1, p}(Z)$ is a sequence such that

$$
\left|\varphi_{1, \lambda}\left(x_{n}\right)\right| \leq M_{2} \text { for some constant } M_{2}>0 \text {, all } n \geq 1 \text { and } m\left(x_{n}\right) \rightarrow 0 \text {. }
$$

We can find $x_{n}^{*} \in \partial \varphi_{1, \lambda}\left(x_{n}\right)$ such that $m\left(x_{n}\right)=\left\|x_{n}^{*}\right\|$ for all $n \geq 1$. We see

$$
x_{n}^{*}=A\left(x_{n}\right)-\lambda\left|x_{n}\right|^{p-2} x_{n}-u_{n}
$$

with $u_{n} \in L^{p^{\prime}}(Z), u_{n}(z) \in \partial j_{1}\left(z, x_{n}(z)\right)$ a.e. on $Z$. Let us show that $\left\{x_{n}\right\} \subset$ $W_{0}^{1, p}(Z)$ is bounded. Arguing indirectly, we assume that $\left\|x_{n}\right\| \rightarrow \infty$ along a relabelled subsequence. Set $y_{n}=\frac{x_{n}}{\left\|x_{n}\right\|}, n \geq 1$. Then at least for a subsequence, we have

$$
y_{n} \stackrel{\mathrm{w}}{\rightarrow} y \text { in } W_{0}^{1, p}(Z), y_{n} \rightarrow y \text { in } L^{p}(Z), y_{n}(z) \rightarrow y(z) \text { a.e. on } Z
$$

and

$$
\left|y_{n}(z)\right| \leq k(z) \text { a.a. } z \in Z \text {, for all } n \geq 1 \text { and with } k \in L^{p}(Z) .
$$

Since $-x_{n}^{-} \in W_{0}^{1, p}(Z)$, we have $\left\langle x_{n}^{*},-x_{n}^{-}\right\rangle \leq \varepsilon_{n}\left\|x_{n}^{-}\right\|$with $\varepsilon_{n} \downarrow 0$, which implies by (3.2) that $\left\|D x_{n}^{-}\right\|_{p}^{p}-\lambda\left\|x_{n}^{-}\right\|_{p}^{p} \leq \varepsilon_{n}\left\|x_{n}^{-}\right\|$. If $\lambda \leq 0$, it is clear that $\left\{x_{n}^{-}\right\} \subset W_{0}^{1, p}(Z)$ is bounded. If $0<\lambda<\lambda_{1}$, we note

$$
\left(1-\frac{\lambda}{\lambda_{1}}\right)\left\|D x_{n}^{-}\right\|_{p}^{p} \leq \varepsilon_{n}\left\|x_{n}^{-}\right\|
$$


so again $\left\{x_{n}^{-}\right\} \subset W_{0}^{1, p}(Z)$ is bounded. Writing $y_{n}=\left(1 /\left\|x_{n}\right\|\right)\left(x_{n}^{+}-x_{n}^{-}\right)$, from the boundedness of $\left\{x_{n}^{-}\right\}$it results that $\left(1 /\left\|x_{n}\right\|\right) x_{n}^{+} \stackrel{\mathrm{w}}{\rightarrow} y$ in $W_{0}^{1, p}(Z)$, and up to a subsequence $\left(1 /\left\|x_{n}\right\|\right) x_{n}^{+}(z) \rightarrow y(z)$ a.e. $z \in Z$, therefore $y \geq 0$.

We now show that $y \neq 0$. To this end we write

$\left.\left|\left\langle A\left(y_{n}\right), y_{n}-y\right\rangle-\lambda \int_{Z}\right| y_{n}\right|^{p-2} y_{n}\left(y_{n}-y\right) d z-\int_{Z} \frac{u_{n}}{\left\|x_{n}\right\|^{p-1}}\left(y_{n}-y\right) d z \mid \leq \varepsilon_{n}\left\|y_{n}-y\right\|$ where we have used $\left\|x_{n}\right\| \geq 1$ for sufficiently large $n$. From (4.1) we infer

$$
\int_{Z}\left|y_{n}\right|^{p-2} y_{n}\left(y_{n}-y\right) d z \rightarrow 0 \text { as } n \rightarrow \infty \text {. }
$$

By virtue of hypothesis $\mathrm{H}(j)_{4}(\mathrm{iii})^{\prime},\left\{\left(1 /\left\|x_{n}\right\|^{p-1}\right) u_{n}\right\}$ is bounded in $L^{p^{\prime}}(Z)$ so

$$
\int_{Z} \frac{u_{n}}{\left\|x_{n}\right\|^{p-1}}\left(y_{n}-y\right) d z \rightarrow 0 \text { as } n \rightarrow \infty \text {. }
$$

Passing to the limit in (4.2) and using the convergences in (4.3) and (4.4), we obtain $\lim _{n \rightarrow \infty}\left\langle A\left(y_{n}\right), y_{n}-y\right\rangle=0$. Then as in the proof of Theorem 3.1 (see Claim 1), we obtain $y_{n} \rightarrow y$ in $W_{0}^{1, p}(Z)$. This entails $\|y\|=1$, hence $y \neq 0, y \geq 0$.

We notice that by hypothesis $\mathrm{H}(j)_{4}(\text { iii })^{\prime}$ we have

$$
\limsup _{x \rightarrow+\infty} \frac{u}{x^{p-1}} \leq c \text { uniformly for almost all } z \in Z \text { and all } u \in \partial j(z, x) .
$$

Given $\varepsilon \in\left(0, \varepsilon_{0}\right)$, with $\varepsilon_{0}$ in $\mathrm{H}(j)_{4}(\mathrm{iv})^{\prime}$, we introduce the set

$$
E_{\varepsilon, n}=\left\{z \in Z: x_{n}(z)>0, \varepsilon_{0}-\varepsilon<\frac{u_{n}(z)}{x_{n}(z)^{p-1}} \leq c+\varepsilon\right\}, \quad \forall n \geq 1 .
$$

Note that for almost all $z \in\{y>0\}$, we have $x_{n}(z) \rightarrow+\infty$. So hypothesis $\mathrm{H}(j)_{4}(\mathrm{iv})^{\prime}$ implies that the characteristic function $\chi_{E_{\varepsilon, n}}$ of $E_{\varepsilon, n}$ has the property $\chi_{E_{\varepsilon, n}}(z) \rightarrow 1$ a.e. on $\{y>0\}$. Assumption $\mathrm{H}(j)_{4}(\text { iii })^{\prime}$ ensures

$$
\frac{\left|u_{n}(z)\right|}{\left\|x_{n}\right\|^{p-1}} \leq \frac{a(z)}{\left\|x_{n}\right\|^{p-1}}+c\left|y_{n}(z)\right|^{p-1} \text { a.e. on } Z \text {. }
$$

Then $\left\{\left(1 /\left\|x_{n}\right\|^{p-1}\right) u_{n}\right\}$ is bounded in $L^{p^{\prime}}(Z)$, so up to a subsequence one has

$$
\frac{1}{\left\|x_{n}\right\|^{p-1}} u_{n} \stackrel{\mathrm{w}}{\rightarrow} h \text { in } L^{p^{\prime}}(Z) \text { as } n \rightarrow \infty, \text { for some } h \in L^{p^{\prime}}(Z) \text {. }
$$

Since using (4.5),

$$
\left(1-\chi_{E_{\varepsilon, n}}\right) \frac{u_{n}}{\left\|x_{n}\right\|^{p-1}} \rightarrow 0 \text { in } L^{1}(\{y>0\})
$$

it follows that

$$
\chi_{E_{\varepsilon, n}} \frac{u_{n}}{\left\|x_{n}\right\|^{p-1}} \stackrel{\mathrm{w}}{\rightarrow} h \text { in } L^{1}(\{y>0\}) \text { as } n \rightarrow \infty .
$$

The definition of the sets $E_{\varepsilon, n}$ shows

$$
\chi_{E_{\varepsilon, n}}(z)\left(\varepsilon_{0}-\varepsilon\right) y_{n}(z)^{p-1} \leq \chi_{E_{\varepsilon, n}}(z) \frac{u_{n}(z)}{\left\|x_{n}\right\|^{p-1}} \leq \chi_{E_{\varepsilon, n}}(z)(c+\varepsilon) y_{n}(z)^{p-1}
$$

for a.e. on $\{y>0\}$. Taking weak limits in $L^{1}(\{y>0\})$, we obtain

$$
\left(\varepsilon_{0}-\varepsilon\right) y(z)^{p-1} \leq h(z) \leq(c+\varepsilon) y(z)^{p-1} \text { a.e. on }\{y>0\} \text {. }
$$


Because $\varepsilon>0$ was arbitrary, we conclude

$$
\varepsilon_{0} y(z)^{p-1} \leq h(z) \leq c y(z)^{p-1} \text { a.e. on }\{y>0\} .
$$

Also from (4.5) it is clear that

$$
h(z)=0 \text { a.e. on }\{y=0\} .
$$

Taking into account that $Z=\{y=0\} \cup\{y>0\}$, from (4.6) and (4.7) we infer

$$
h(z)=g(z) y(z)^{p-1} \text { with } \varepsilon_{0} \leq g(z) \leq c \text { a.e. on } Z .
$$

The choice of the sequence $\left\{x_{n}\right\} \subset W_{0}^{1, p}(Z)$ implies

$$
\left.\left|\left\langle A\left(y_{n}\right), v\right\rangle-\lambda \int_{Z}\right| y_{n}\right|^{p-2} y_{n} v d z-\int_{Z} \frac{u_{n}}{\left\|x_{n}\right\|^{p-1}} v d z \mid \leq \varepsilon_{n}\|v\|, \quad \forall v \in W_{0}^{1, p}(Z),
$$

with $\varepsilon_{n} \downarrow 0$. Using $y_{n} \rightarrow y$ in $W_{0}^{1, p}(Z)$ and (4.8), we obtain that in $W^{-1, p^{\prime}}(Z)$ there holds

$$
-\operatorname{div}\left(\|D y(z)\|^{p-2} D y(z)\right)=(\lambda+g(z))|y(z)|^{p-2} y(z) .
$$

Exploiting (4.8), the assumption $\lambda>\lambda_{1}-\varepsilon_{0}$ and the decreasing monotonicity of the principal eigenvalue $\lambda_{1}(\hat{g})>0$ of the weighted eigenvalue problem $-\Delta_{p} v(z)=$ $\lambda \hat{g}(z)|v(z)|^{p-2} v(z)$ a.e. on $Z,\left.v\right|_{\partial Z}=0$, on the weight function $\hat{g} \in L^{\infty}(Z)_{+}$, we deduce

$$
\lambda_{1}(g+\lambda)<\lambda_{1}\left(\lambda_{1}\right)=1 .
$$

By (4.9) in conjunction with $y \neq 0$, we get that $y$ is an eigenfunction whose corresponding eigenvalue is 1 for the problem with weight $g+\lambda \in L^{\infty}(Z)_{+}$. According to (4.10), $y$ cannot be a principal eigenfunction that is associated to the first eigenvalue $\lambda_{1}(g+\lambda)$. Knowing that only the principal eigenfunctions have constant sign (see Anane [2]), we deduce that $y$ must change sign, thus achieving a contradiction with $y \geq 0$. This proves that $\left\{x_{n}\right\} \subset W_{0}^{1, p}(Z)$ is bounded, from which as in Claim 1 of the proof of Theorem 3.1 we conclude that $\varphi_{1, \lambda}$ satisfies the nonsmooth PS-condition.

Claim 2. For every $\lambda \in\left(\lambda_{1}-\varepsilon_{0}, \lambda_{1}\right)$, we can find $v_{\lambda} \in W_{0}^{1, p}(Z)$ of arbitrarily large norm such that $\varphi_{1, \lambda}\left(v_{\lambda}\right)<0=\varphi_{1, \lambda}(0)$.

Fix $\lambda \in\left(\lambda_{1}-\varepsilon_{0}, \lambda_{1}\right)$. For a.a. $z \in Z$ and all $s \in \mathbb{R} \backslash D(z)$ with $|D(z)|_{1}=0$ there exists $\frac{d}{d s} j(z, s)$ and $\frac{d}{d s} j(z, s) \in \partial j(z, s)$. So by $\mathrm{H}(j)_{4}(\text { iv })^{\prime}$ for $\varepsilon \in\left(0, \varepsilon_{0}\right)$, there is $M=M(\varepsilon)>0$ such that

$$
\frac{d}{d s} j(z, s) \geq\left(\varepsilon_{0}-\varepsilon\right) s^{p-1} \text { for a.a. } z \in Z, \forall s \geq M, s \notin D(z) .
$$

By $\mathrm{H}(j)_{4}(\mathrm{iii})^{\prime}$ and (4.11), it is allowed to write

$$
\begin{aligned}
j(z, x) & =\int_{0}^{x} \frac{d}{d s} j(z, s) d s=\int_{0}^{M} \frac{d}{d s} j(z, s) d s+\int_{M}^{x} \frac{d}{d s} j(z, s) d s \\
& \geq-\xi_{\varepsilon}+\frac{1}{p}\left(\varepsilon_{0}-\varepsilon\right)\left(x^{p}-M^{p}\right) \text { for a.a. } z \in Z, \forall x \geq M,
\end{aligned}
$$

for some constant $\xi_{\varepsilon}>0$. Thus there is $c_{\varepsilon}>0$ such that

$$
j_{1}(z, x)=j(z, x) \geq \frac{1}{p}\left(\varepsilon_{0}-\varepsilon\right) x^{p}-c_{\varepsilon} \text { for a.a. } z \in Z, \forall x \geq 0 .
$$


Then, from (3.3), (2.2) and (4.12), for all $t>0$ we get with some constant $\hat{c}_{\varepsilon}>0$ that

$$
\varphi_{1, \lambda}\left(t u_{1}\right) \leq \frac{t^{p}}{p}\left(1-\frac{\lambda+\varepsilon_{0}-\varepsilon}{\lambda_{1}}\right)\left\|D u_{1}\right\|_{p}^{p}+\hat{c}_{\varepsilon} .
$$

Choosing $\varepsilon<\lambda-\lambda_{1}+\varepsilon_{0}$, we obtain that $\varphi_{1, \lambda}\left(t u_{1}\right) \rightarrow-\infty$ as $t \rightarrow+\infty$, which establishes Claim 2.

Using $\mathrm{H}(j)_{1}(\mathrm{v})$, as in Claim 2 of the proof of Theorem 3.1 we find $\rho>0$ with

$$
\inf \left\{\varphi_{1, \lambda}(x):\|x\|=\rho\right\}=\beta>0 .
$$

Claims 1, 2 and assertion (4.13) enable us to make use of Theorem 2.1, which gives for all $\lambda \in\left(\lambda_{1}-\varepsilon_{0}, \lambda_{1}\right)$ the existence of an $x=x(\lambda) \in W_{0}^{1, p}(Z)$ such that $\varphi_{1, \lambda}(x) \geq \beta>0=\varphi_{1, \lambda}(0)$ (thus $x \neq 0$ ) and $0 \in \partial \varphi_{1, \lambda}(x)$. The reasoning in the final part of the proof of Theorem 3.1, based on hypothesis $\mathrm{H}(j)_{1}$ (vi), allows us to conclude that $x$ is a positive solution of problem (1.1).

Example 4.2. Consider problem (1.1) with the locally Lipschitz potential $j(z, x)=$ $j(x)$ given by

$$
j(x)=\min \left\{\frac{1}{p}|x|^{p}, \frac{1}{r}|x|^{r}\right\}, \text { with } 1<p<r<p^{*} .
$$

A direct verification shows that the assumptions of Theorem 4.1 are fulfilled. We obtain the existence of a positive solution to problem (1.1) with $j(z, x)=j(x)$ as above and $\lambda \in\left(\lambda_{1}-1, \lambda_{1}\right)$.

\section{Multiple SOlutions With NEAR RESONANCE FROM The Right}

We now examine the eigenvalue problem (1.1) near resonance from the right of $\lambda_{1}$. The hypotheses on the nonsmooth potential $j(z, x)$ are the following:

$\mathrm{H}(j)_{5} \quad j: Z \times \mathbb{R} \rightarrow \mathbb{R}$ is a function such that $j(z, 0)=0$ a.e. on $Z$, it satisfies $\mathrm{H}(j)_{1}(\mathrm{i})-(\mathrm{iii})$, and

(iv) $)^{\prime \prime}$ there exists $\hat{\eta} \in L^{\infty}(Z)$ such that $\hat{\eta}(z) \leq 0$ a.e. on $Z$ with strict inequality on a set of positive measure and

$$
\limsup _{|x| \rightarrow+\infty} \frac{p j(z, x)}{|x|^{p}} \leq \hat{\eta}(z) \text { uniformly for almost all } z \in Z \text {; }
$$

$(\mathrm{v})^{\prime \prime}$ there exists $\delta>0$ such that for almost all $z \in Z$ and all $0 \leq x \leq \delta$ or $-\delta \leq x \leq 0$, we have $j(z, x) \geq 0$.

Theorem 5.1. If hypotheses $\mathrm{H}(j)_{5}$ hold, then there exists $\hat{\varepsilon}>0$ such that for all $\lambda \in\left(\lambda_{1}, \lambda_{1}+\hat{\varepsilon}\right)$ problem (1.1) has a nontrivial solution $x \in C_{0}^{1}(\bar{Z})$.

Proof. Suppose that $\lambda=\lambda_{1}+\varepsilon$ with $\varepsilon>0$. From hypotheses $\mathrm{H}(j)_{1}(\mathrm{iii}), \mathrm{H}(j)_{5}(\mathrm{iv})^{\prime \prime}$ and the mean value theorem for locally Lipschitz functions, we can find a constant $c_{\varepsilon}>0$ such that

$$
j(z, x) \leq \frac{1}{p}(\hat{\eta}(z)+\varepsilon)|x|^{p}+c_{\varepsilon} \text { for a.a. } z \in Z, \forall x \in \mathbb{R} .
$$

Consider the locally Lipschitz functional $\varphi_{\lambda}: W_{0}^{1, p}(Z) \rightarrow \mathbb{R}$ defined by

$$
\varphi_{\lambda}(x)=\frac{1}{p}\|D x\|_{p}^{p}-\frac{\lambda}{p}\|x\|_{p}^{p}-\int_{Z} j(z, x(z)) d z, \quad \forall x \in W_{0}^{1, p}(Z) .
$$


By (5.2), (5.1) and Lemma 3.4 we derive the estimate

$$
\begin{aligned}
\varphi_{\lambda}(x) & \geq \frac{1}{p}\|D x\|_{p}^{p}-\frac{1}{p} \int_{Z}\left(\lambda_{1}+\hat{\eta}(z)\right)|x(z)|^{p} d z-\frac{2 \varepsilon}{p}\|x\|_{p}^{p}-\hat{c}_{\varepsilon} \\
& \geq \frac{1}{p}\left(\xi_{0}-\frac{2 \varepsilon}{\lambda_{1}}\right)\|D x\|_{p}^{p}-\hat{c}_{\varepsilon}, \quad \forall x \in W_{0}^{1, p}(Z),
\end{aligned}
$$

with a constant $\hat{c}_{\varepsilon}>0$ and where $\xi_{0}>0$ is independent of $\varepsilon>0$. We see that if $\varepsilon \in$ $(0, \hat{\varepsilon})$ with $\hat{\varepsilon}=\frac{1}{2} \xi_{0} \lambda_{1}$, then $\varphi_{\lambda}$ is coercive, and being weakly lower semicontinuous, we obtain $x=x(\lambda) \in W_{0}^{1, p}(Z)$ such that

$$
\varphi_{\lambda}(x)=\inf _{W_{0}^{1, p}(Z)} \varphi_{\lambda} .
$$

Therefore $x=x(\lambda)$ solves problem (1.1). We claim

$$
\varphi_{\lambda}(x)<0=\varphi_{\lambda}(0) .
$$

Suppose that the first option in hypothesis $\mathrm{H}(j)_{5}(\mathrm{v})^{\prime \prime}$ is valid (the reasoning is similar in the other case). Since $u_{1} \in C_{0}^{1}(\bar{Z})$, we can find $t>0$ such that $\left\|t u_{1}\right\|_{\infty} \leq$ $\delta$. Then, by (5.2) and $\mathrm{H}(j)_{5}(\mathrm{v})^{\prime \prime}$, we have

$$
\varphi_{\lambda}\left(t u_{1}\right) \leq \frac{t^{p}}{p}\left(1-\frac{\lambda}{\lambda_{1}}\right)\left\|D u_{1}\right\|_{p}^{p}<0 .
$$

The claim is verified and so $x \neq 0$. From nonlinear regularity theory we obtain that $x \in C_{0}^{1}(\bar{Z})$, thus completing the proof.

Example 5.2. Consider problem (1.1) with

$$
j(z, x)= \begin{cases}|x| & \text { if }|x| \leq 1 \\ |x|+\hat{\eta}(z)(|x|-1)^{p} & \text { if }|x| \geq 1\end{cases}
$$

where $\hat{\eta} \in L^{\infty}(Z)$ is such that $\hat{\eta}(z) \leq 0$ a.e. on $Z$ with strict inequality on a set of positive measure. One can easily check that hypotheses $\mathrm{H}(j)_{5}$ are satisfied and so Theorem 5.1 applies to problem (1.1) with $j(z, x)$ as defined above and $\lambda$ near $\lambda_{1}$ from the right.

Finally, strengthening hypothesis $\mathrm{H}(j)_{5}(\mathrm{v})^{\prime \prime}$ we prove a theorem on the existence of multiple nontrivial solutions for problem (1.1) under near resonance at $\lambda_{1}>0$ from the right. Consider the direct sum decomposition

$$
W_{0}^{1, p}(Z)=\mathbb{R} u_{1} \oplus V
$$

with $V=\left\{v \in W_{0}^{1, p}(Z): \int_{Z} u_{1}^{p-1} v d z=0\right\}$. Since $\lambda_{1}>0$ is isolated, there is $\hat{\lambda}_{2}>\lambda_{1}$ such that

$$
\hat{\lambda}_{2}\|v\|_{p}^{p} \leq\|D v\|_{p}^{p}, \quad \forall v \in V .
$$

We formulate the assumptions

$\mathrm{H}(j)_{6} \quad j: Z \times \mathbb{R} \rightarrow \mathbb{R}$ is a function such that $j(z, 0)=0$ a.e. on $Z$, it satisfies $\mathrm{H}(j)_{1}(\mathrm{i})-(\mathrm{iii}), \mathrm{H}(j)_{5}(\mathrm{iv})^{\prime \prime}$ and

$(\mathrm{v})^{\prime \prime \prime}$ there exists $\delta>0$ such that for almost all $z \in Z$ and all $x \in[-\delta, \delta]$, one has

$$
0 \leq j(z, x) \leq \frac{\beta}{p}|x|^{p} \text { with } \beta<\hat{\lambda}_{2}-\lambda_{1} .
$$

Theorem 5.3. If hypotheses $\mathrm{H}(j)_{6}$ hold, then there exists $\hat{\varepsilon}_{0}>0$ such that for all $\lambda \in\left(\lambda_{1}, \lambda_{1}+\hat{\varepsilon}_{0}\right)$ problem (1.1) has at least two nontrivial solutions $x_{1}, x_{2} \in C_{0}^{1}(\bar{Z})$. 
Proof. In the proof of Theorem 5.1 we obtained an $\hat{\varepsilon}>0$ such that for all $\varepsilon \in(0, \hat{\varepsilon})$, the functional $\varphi_{\lambda}$ in (5.2) is coercive with $\lambda=\lambda_{1}+\varepsilon$. Therefore it is bounded below and satisfies the nonsmooth PS-condition. Moreover, again in the proof of Theorem 5.1 , it was established that $\inf _{W_{0}^{1, p}(Z)} \varphi_{\lambda}<\varphi_{\lambda}(0)=0$.

Since $u_{1} \in C_{0}^{1}(\bar{Z})$, we can find $t_{0}>0$ such that if $|t| \leq t_{0}$, then $\left|t u_{1}(z)\right| \leq \delta$ for all $z \in Z$, with $\delta>0$ determined in hypothesis $\mathrm{H}(j)_{6}(\mathrm{v})^{\prime \prime \prime}$. By $(5.2), \mathrm{H}(j)_{6}(\mathrm{v})^{\prime \prime \prime}$ and since $\lambda>\lambda_{1}$, we get

$$
\varphi_{\lambda}\left(t u_{1}\right) \leq \frac{t^{p}}{p}\left(1-\frac{\lambda}{\lambda_{1}}\right)\left\|D u_{1}\right\|_{p}^{p} \leq 0 \quad \text { for }|t| \leq t_{0} .
$$

On the other hand, from hypotheses $\mathrm{H}(j)_{1}$ (iii) and $\mathrm{H}(j)_{6}(\mathrm{v})^{\prime \prime \prime}$ we have

$$
j(z, x) \leq \frac{\beta}{p}|x|^{p}+\hat{c}|x|^{r} \text { for a.a. } z \in Z, \forall x \in \mathbb{R},
$$

with a constant $\hat{c}>0$. Then from (5.2)-(5.4), (5.6) we obtain

$$
\varphi_{\lambda}(v) \geq \frac{1}{p}\left(1-\frac{\lambda_{1}+\varepsilon+\beta}{\hat{\lambda}_{2}}\right)\|D v\|_{p}^{p}-\hat{c}_{1}\|D v\|_{p}^{r}, \quad \forall v \in V, \lambda=\lambda_{1}+\varepsilon, \varepsilon>0,
$$

for a new constant $\hat{c}_{1}>0$. Since $\hat{\lambda}_{2}-\lambda_{1}-\beta>0$ (cf. hypothesis $\left.\mathrm{H}(j)_{6}(\mathrm{v})^{\prime \prime \prime}\right)$, we may take $\varepsilon \in\left(0, \hat{\lambda}_{2}-\lambda_{1}-\beta\right)$. Hence by (5.7), recalling $r>p$, we can find $\hat{\delta}>0$ such that

$$
\varphi_{\lambda}(v) \geq 0 \text { for all } v \in V \text { with }\|v\| \leq \hat{\delta} .
$$

So if $\hat{\varepsilon}_{0}=\min \left\{\hat{\varepsilon}, \hat{\lambda}_{2}-\lambda_{1}-\beta\right\}$, then due to (5.5), (5.8), we can apply Theorem 2.2 that provides two nontrivial critical points of $\varphi_{\lambda}$. This amounts to saying that there exist two nontrivial solutions of problem (1.1). Through the nonlinear regularity theory, they belong to $C_{0}^{1}(\bar{Z})$.

Example 5.4. Consider problem (1.1) with the locally Lipschitz potential $j(z, x)=$ $j(x)$ introduced as follows:

$$
j(x)= \begin{cases}\frac{\beta}{p} \ln \left(|x|^{p}+1\right) & \text { if }|x| \leq 1, \\ (2-|x|) \frac{\beta}{p} \ln \left(|x|^{p}+1\right)+(|x|-1) \frac{\hat{\eta}(z)}{p}|x|^{p} & \text { if } 1<|x|<2, \\ \frac{\hat{\eta}(z)}{p}|x|^{p} & \text { if }|x| \geq 2 .\end{cases}
$$

Here $\hat{\eta} \in L^{\infty}(Z)$ is such that $\hat{\eta}(z) \leq 0$ a.e. on $Z$ with strict inequality on a set of positive measure and $0 \leq \beta<\hat{\lambda}_{2}-\lambda_{1}$. It is easily seen that hypotheses $\mathrm{H}(j)_{6}$ are verified. Hence Theorem 5.3 can be applied to problem (1.1) with the above potential $j(z, x)=j(x)$ in the case of near resonance from the right at $\lambda_{1}$.

\section{REFERENCES}

1. A. Ambrosetti and P.H. Rabinowitz, Dual variational methods in critical point theory and applications, J. Funct. Anal. 14 (1973), 349-381. MR0370183 (51:6412)

2. A. Anane, Simplicité et isolation de la première valeur propre du $p$-Laplacien avec poids, $C$. R. Acad. Sci. Paris Sér. I Math. 305 (1987), 725-728. MR920052 (89e:35124)

3. G. Barletta and S.A. Marano, Some remarks on critical point theory for locally Lipschitz functions, Glasg. Math. J. 45 (2003), 131-141. MR1972703 (2004e:58016) 
4. K.-C. Chang, Variational methods for nondifferentiable functionals and their applications to partial differential equations, J. Math. Anal. Appl. 80 (1981), 102-129. MR614246 (82h:35025)

5. F.H. Clarke, Optimization and Nonsmooth Analysis, John Wiley and Sons, New York, 1983. MR709590 (85m:49002)

6. L. Gasiński and N.S. Papageorgiou, Multiple solutions for semilinear hemivariational inequalities at resonance, Publ. Math. Debrecen 59 (2001), 121-146. MR1853497 (2003d:35202)

7. L. Gasiński and N.S. Papageorgiou, Existence of solutions and of multiple solutions for eigenvalue problems of hemivariational inequalities, Adv. Math. Sci. Appl. 11 (2001), 437-464. MR1842386 (2002c:49014)

8. L. Gasiński and N.S. Papageorgiou, Nonsmooth Critical Point Theory and Nonlinear Boundary Value Problems, Chapman \& Hall/CRC, Boca Raton, FL, 2005. MR2092433 (2006f:58013)

9. D. Goeleven, D. Motreanu, and P.D. Panagiotopoulos, Multiple solutions for a class of eigenvalue problems in hemivariational inequalities, Nonlinear Anal. 29 (1997), 9-26. MR1447566 (98f:47072)

10. D. Goeleven, D. Motreanu, and P.D. Panagiotopoulos, Eigenvalue problems for variationalhemivariational inequalities at resonance, Nonlinear Anal. 33 (1998), 161-180. MR1621101 (99b:47094)

11. G. Li and H.-S. Zhou, Asymptotically linear Dirichlet problem for the p-Laplacian, Nonlinear Anal. 43 (2001), 1043-1055. MR1812073 (2001m:35113)

12. R. Livrea, S.A. Marano, and D. Motreanu, Critical points for nondifferentiable functions in presence of splitting, J. Differential Equations 226 (2006), 704-725. MR2237697

13. D. Motreanu and P.D. Panagiotopoulos, A minimax approach to the eigenvalue problem of hemivariational inequalities and applications, Appl. Anal. 58 (1995), 53-76. MR1384589 (97h:47064)

14. D. Motreanu and P.D. Panagiotopoulos, On the eigenvalue problem for hemivariational inequalities: existence and multiplicity of solutions, J. Math. Anal. Appl. 197 (1996), 75-89. MR1371277 (96k:47113)

15. D. Motreanu and P.D. Panagiotopoulos, Minimax Theorems and Qualitative Properties of the Solutions of Hemivariational Inequalities, Kluwer Academic Publishers, Dordrecht, 1999. MR1675895 (2000a:49015)

16. D. Motreanu and N.S. Papageorgiou, Multiple solutions for nonlinear elliptic equations at resonance with a nonsmooth potential, Nonlinear Anal. 56 (2004), 1211-1234. MR2040681 (2005b:35087)

17. Z. Naniewicz and P.D. Panagiotopoulos, Mathematical Theory of Hemivariational Inequalities and Applications, Marcel Dekker, Inc., New York, 1995. MR1304257 (96d:47067)

18. J. L. Vázquez, A strong maximum principle for some quasilinear elliptic equations, Appl. Math. Optimization 12 (1984), 191-202. MR768629 (86m:35018)

19. X. Wu, A new critical point theorem for locally Lipschitz functionals with applications to differential equations, Nonlinear Anal. 66 (2007), 624-638. MR2274873 (2007g:35068)

Département de Mathématiques, Université de Perpignan, 66860 Perpignan, France E-mail address: motreanu@univ-perp.fr

Département de Mathématiques, Université de Perpignan, 66860 Perpignan, France E-mail address: viorica@univ-perp.fr

Department of Mathematics, National Technical University, Zografou Campus, Athens 15780, Greece

E-mail address: npapg@math.ntua.gr 